\title{
Binding energies of excitonic complexes in type-II quantum rings from diffusion quantum Monte Carlo calculations
}

\author{
D. M. Thomas ${ }^{*}$ R. J. Hunt, N. D. Drummond, and M. Hayne \\ Department of Physics, Lancaster University, Lancaster LA1 4YB, United Kingdom
}

(Dated: February 22, 2019)

\begin{abstract}
Excitonic complexes in type-II quantum-ring heterostructures may be considered as artificial atoms due to the confinement of only one charge-carrier type in an artificial nucleus. Binding energies of excitons, trions, and biexcitons in these nanostructures are then effectively ionization energies of these artificial atoms. The binding energies reported here are calculated within the effective-mass approximation using the diffusion quantum Monte Carlo method and realistic geometries for gallium antimonide rings in gallium arsenide. The electrons form a halo outside the ring, with very little charge density inside the central cavity of the ring. The de-excitonization and binding energies of the complexes are relatively independent of the precise shape of the ring.

PACS numbers: 71.35.-y, 71.35.Pq, 81.07.Ta, 81.05.Ea, 02.70.Ss
\end{abstract}

\section{INTRODUCTION}

Quantum-dot and quantum-ring heterostructures have long been hailed as "artificial atoms" [1-4 due to their ability to confine charge carriers in all three spatial dimensions. Material combinations exhibiting type-I band alignment produce nanostructures in which both electrons and holes are confined to the same spatial region, and such nanostructures have been studied extensively over the last two decades [5] 7]. In type-II nanostructures, on the other hand, only holes but not electrons (or vice versa) are confined, presenting a rich variety of new physics [8] 10. For example, GaSb quantum dots or quantum rings in GaAs provide very deep confining potentials for holes [11, while strain in the GaSb raises the conduction-band minimum, expelling the electrons 12. Excitonic complexes in type-II nanostructures are in fact very much more like artificial atoms than is the case for type-I nanostructures, because the electrons are bound to the holes in the "artificial nuclei" purely by the Coulomb interaction, rather than being confined themselves. Type-II quantum rings are an intriguingly distinct type of artificial atom with no natural analog due to the radical difference between the ring-shaped "artificial nucleus" and the pointlike nucleus of a real atom.

Excitons in type-II quantum dots have been extensively studied both experimentally [13-16] and theoretically [11, 17, 18; however, while there has been some experimental work on carrier complexes in typeII quantum-ring nanostructures [19-22, there has been little theoretical work to date. The spatial separation of charge carriers allows for a variety of interesting optoelectronic properties [19, 23, including extended recombination times, making type-II quantum rings ideal candidates for applications such as memory devices 24] and solar cells [16]. Binding energies of excitonic complexes reported here are effectively ionization energies of

* d.thomas4@lancaster.ac.uk these artificial atoms. GaSb quantum rings in GaAs may be produced by molecular beam epitaxy [20, 22, 23, 25] and can form with a variety of different cross-sections ranging from triangular, to semicircular [20, and even trapezoidal 23. These quantum rings exhibit type-II behavior, with the holes strongly confined to the rings. Scanning tunneling microscopy (STM) has been used to investigate the shape and size of the GaSb rings, and their optical properties have been studied in photoluminescence experiments [1922].

In this work we solve an effective-mass model of excitons $(\mathrm{X})$, positive and negative trions $\left(\mathrm{X}^{+}\right.$and $\left.\mathrm{X}^{-}\right)$, and biexcitons (XX) in type-II quantum-ring heterostructures, focusing on GaSb rings in GaAs. The holes are confined to the ring, which is modeled as an infinite potential well, while the electrons are excluded from the ring but bound to the holes by an isotropically screened Coulomb interaction. The kinetic energy of the tightly confined holes is the dominant contribution to the total energy of each complex; however, the electron-hole attraction is non-negligible, as is the hole-hole repulsion. The ring was chosen to have a rectangular cross-section for computational convenience. The ring is centered on the origin, orientated so that the axis of rotation is the $z$ axis and the midpoint in the $z$ direction is the $x-y$ plane. The three parameters defining the ring geometry are the half height of the ring $R_{z}$, the inner radius of the ring $r_{\mathrm{i}}$, and the outer radius $r_{\mathrm{o}}$. In our model the electron and hole densities do not overlap, so we cannot estimate recombination rates; however our model is reasonable for calculating binding energies.

Energies are given in units of the exciton Rydberg $R_{\mathrm{y}}^{*}=\mu e^{4} /\left[2(4 \pi \epsilon)^{2} \hbar^{2}\right]$ and lengths in units of the exciton Bohr radius $a_{0}^{*}=4 \pi \epsilon \hbar^{2} /\left(\mu e^{2}\right)$, where $\epsilon$ is the permittivity of the medium, $\hbar$ is the Dirac constant, and $e$ is the magnitude of the electron charge. The electron-hole reduced mass is $\mu=m_{\mathrm{e}}^{*} m_{\mathrm{h}}^{*} /\left(m_{\mathrm{e}}^{*}+m_{\mathrm{h}}^{*}\right)$, where $m_{\mathrm{e}}^{*}$ and $m_{\mathrm{h}}^{*}$ are the effective masses of an electron and a hole, respectively. Within the effective-mass approximation the 
Hamiltonian for a biexciton is

$$
\begin{aligned}
\frac{\hat{H}}{2 R_{\mathrm{y}}^{*}}= & -\frac{\left(a_{0}^{*}\right)^{2} \mu}{2 m_{\mathrm{e}}^{*}}\left(\nabla_{\mathrm{e}_{1}}^{2}+\nabla_{\mathrm{e}_{2}}^{2}\right)-\frac{\left(a_{0}^{*}\right)^{2} \mu}{2 m_{\mathrm{h}}^{*}}\left(\nabla_{\mathrm{h}_{1}}^{2}+\nabla_{\mathrm{h}_{2}}^{2}\right) \\
& +\frac{a_{0}^{*}}{r_{\mathrm{e}_{1} \mathrm{e}_{2}}}+\frac{a_{0}^{*}}{r_{\mathrm{h}_{1} \mathrm{~h}_{2}}}-\frac{a_{0}^{*}}{r_{\mathrm{e}_{1} \mathrm{~h}_{1}}}-\frac{a_{0}^{*}}{r_{\mathrm{e}_{1} \mathrm{~h}_{2}}}-\frac{a_{0}^{*}}{r_{\mathrm{e}_{2} \mathrm{~h}_{1}}}-\frac{a_{0}^{*}}{r_{\mathrm{e}_{2} \mathrm{~h}_{2}}} \\
& +\sum_{i} V_{i}\left(\frac{\mathbf{r}_{i}}{a_{0}^{*}}\right)
\end{aligned}
$$

in excitonic units, where $r_{i j}=\left|\mathbf{r}_{i}-\mathbf{r}_{j}\right|$ and $V_{i}$ is the confining potential, which is infinite inside the ring and zero outside for electrons, and vice versa for holes. This is an inhomogeneous four-body problem, but the diffusion quantum Monte Carlo (DMC) method [26, 27] can be used to calculate the exact ground-state energy for each complex, and hence the de-excitonization and binding energies. The trion and biexciton de-excitonization energies $E_{\mathrm{D}}^{\mathrm{X}^{ \pm}}$and $E_{\mathrm{D}}^{\mathrm{XX}}$ are $E_{\mathrm{D}}^{\mathrm{X}^{-}}=E^{\mathrm{X}}-E^{\mathrm{X}^{-}}, E_{\mathrm{D}}^{\mathrm{X}^{+}}=$ $E^{\mathrm{X}}+E^{\mathrm{h}^{+}}-E^{\mathrm{X}^{+}}$, and $E_{\mathrm{D}}^{\mathrm{XX}}=2 E^{\mathrm{X}}-E^{\mathrm{XX}}$, where $E^{i}$ is the ground-state total energy for complex $i$. These are the energies at which trion and biexciton peaks are expected to appear relative to the exciton peak in the photoluminescence spectrum of a quantum ring [21. The sign is such that for a free trion or biexciton $E_{\mathrm{D}}>0$. The binding energies - the energy difference between a complex and its most energetically favorable daughter products, bearing in mind that the holes are confined to the ring-are $E_{\mathrm{b}}^{\mathrm{X}}=E^{\mathrm{X}}-E^{\mathrm{h}^{+}}, E_{\mathrm{b}}^{\mathrm{X}^{-}}=E^{\mathrm{X}^{-}}-E^{\mathrm{X}}$, $E_{\mathrm{b}}^{\mathrm{X}^{+}}=E^{\mathrm{X}^{+}}-E^{2 \mathrm{~h}^{+}}$, and $E_{\mathrm{b}}^{\mathrm{XX}}=E^{\mathrm{XX}}-E^{\mathrm{X}^{+}}$, where $E^{2 \mathrm{~h}^{+}}$is the energy of two holes confined to the same ring. The binding energy determines the temperature at which a complex becomes unstable against dissociation into smaller complexes.

\section{COMPUTATIONAL METHODOLOGY}

The CASINO code 28] was used to perform DMC calculations of the ground-state energies of excitons, trions, and biexcitons in quantum-ring heterostructures. DMC is a stochastic projection method that finds the groundstate component of a trial wave function. In this work the trial wave function was optimized using the variational Monte Carlo (VMC) method, in which many-body expectation values are evaluated using Monte Carlo integration. The trial wave function $\Psi_{\mathrm{T}}$ was of Slater-Jastrow form; e.g., for the biexciton:

$$
\Psi_{\mathrm{T}}(\mathbf{R})=\exp [J(\mathbf{R})] \phi_{\mathrm{e}}\left(\mathbf{r}_{\mathrm{e}_{1}}\right) \phi_{\mathrm{e}}\left(\mathbf{r}_{\mathrm{e}_{2}}\right) \phi_{\mathrm{h}}\left(\mathbf{r}_{\mathrm{h}_{1}}\right) \phi_{\mathrm{h}}\left(\mathbf{r}_{\mathrm{h}_{2}}\right),
$$

where $\mathbf{R}=\left(\mathbf{r}_{\mathrm{e}_{1}}, \mathbf{r}_{\mathrm{e}_{2}}, \mathbf{r}_{\mathrm{h}_{1}}, \mathbf{r}_{\mathrm{h}_{2}}\right)$. The hole orbital $\phi_{\mathrm{h}}$ was taken to be the exact ground-state solution to the Schrödinger equation for a single hole confined to the ring:

$$
\phi_{\mathrm{h}}(\mathbf{r})=\left[\frac{-J_{0}(\beta r) Y_{0}\left(\beta r_{\mathrm{i}}\right)}{J_{0}\left(\beta r_{\mathrm{i}}\right)}+Y_{0}(\beta r)\right] \cos \left(\frac{\pi z}{2 R_{z}}\right),
$$

where $J_{0}$ and $Y_{0}$ are Bessel functions of the first and second kind, respectively. The constant $\beta$ is determined by imposing the boundary condition $\phi_{\mathrm{h}}\left(r_{\mathrm{o}}\right)=0$ numerically for each ring size using the Newton-Raphson method; the other boundary conditions are already satisfied by Eq. (3). The electronic behavior is dominated by Coulomb attraction to the positively charged ring together with hard-wall repulsion from the boundary of the ring. At short range the electron orbital $\phi_{\mathrm{e}}$ linearly decreases to zero on the ring boundary, while at long range the electron orbital decays exponentially to keep the electrons localized to the ring; i.e., the behavior is hydrogenic at long range. The electron orbital $\phi_{\mathrm{e}}$ enforces the correct long- and short-range behavior, with the mid-range behavior determined by the Jastrow factor and variational freedom in the electron orbital. The electron orbital was formed piecewise in eight regions about the ring, with the functions in each region being matched at the boundaries to ensure the value and gradient were smooth everywhere and the orbital was zero inside the ring. See the Supplemental Material for the full form of the orbital. The Jastrow exponent $J(\mathbf{R})$ included a pairwise sum of terms of the form $u_{i j}(r)= \pm \mu_{i j} r /\left(1+c_{i j} r\right)$ for each pair of particles $i$ and $j$ separated by distance $r$ with reduced mass $\mu_{i j}$. The + sign was used for particles with the same charge and - for particles with opposite charge. $c_{i j}$ is a variational parameter, which was different for each particle-pair type. This form ensured the Kato cusp conditions were satisfied [29]. Other one-, two-, and three-body polynomial terms were also included in the Jastrow exponent; these were smoothly truncated at finite range [30, 31. VMC energy minimization was used to optimize the trial wave functions 32 . The fixed-node DMC algorithm is exact for the groundstate energy of an exciton, trion, or biexciton, because of the distinguishability of the particles, which leads to a nodeless wave function. Pairs of DMC calculations were performed with time steps in a $1: 4$ ratio and target configuration populations in a $4: 1$ ratio and the results were extrapolated linearly to zero time step and infinite population. Charge densities were obtained by binning the radial and axial coordinates of each of the particles sampled during VMC and DMC calculations, cylindrically averaging, and then calculating the extrapolated estimate. The errors in the VMC and DMC estimates of the charge density $\left(\rho_{\mathrm{VMC}}\right.$ and $\left.\rho_{\mathrm{DMC}}\right)$ are linear in the error in the trial wave function; however, the error in the extrapolated estimate $2 \rho_{\mathrm{DMC}}-\rho_{\mathrm{VMC}}$ is quadratic in the error in the trial wave function [27].

\section{RESULTS AND DISCUSSION}

All energies and charge densities are reported for a ring composed of GaSb surrounded by GaAs. The electron and hole masses are taken to be $m_{\mathrm{e}}^{*}=0.063 m_{\mathrm{e}}$ and $m_{\mathrm{h}}^{*}=$ $0.4 m_{\mathrm{e}}$, respectively, where $m_{\mathrm{e}}$ is the bare electron mass. The former is the effective mass of an electron in bulk 
GaAs and the latter is the mass of a heavy hole in bulk GaSb [33]. The permittivity is taken to be $\epsilon=12.9 \epsilon_{0}$, where $\epsilon_{0}$ is the permittivity of free space. This is the permittivity of bulk GaAs [33. Data from Ref. 20] were used to obtain experimentally relevant values for the ring size; these values were $R_{z}=2.5 \mathrm{~nm}=0.199 a_{0}^{*}, r_{\mathrm{i}}=6 \mathrm{~nm}$ $=0.479 a_{0}^{*}$, and $r_{\mathrm{o}}=10 \mathrm{~nm}=0.799 a_{0}^{*}$. This geometry was used as the starting point for our calculations; the aspect ratio $2 R_{z} /\left(r_{\mathrm{o}}-r_{\mathrm{i}}\right)$ of the ring was then varied subject to the constraints that the volume of the ring $2 \pi R_{z}\left(r_{\mathrm{o}}^{2}-r_{\mathrm{i}}^{2}\right)$ was constant and the center of the ring in the radial direction $\left(r_{\mathrm{i}}+r_{\mathrm{o}}\right) / 2$ was fixed. A ring with aspect ratio much less than 1 is akin to a thin disc with a hole in the center, while a ring with aspect ratio much greater than 1 resembles a pipe.

The analytically evaluated variation in the hole energy against aspect ratio is shown in Fig. 11. The minimum energy occurs when the cross-section is square; away from the minimum, the energy goes roughly as $1 / L^{2}$, where $L=\min \left\{2 R_{z}, r_{\mathrm{o}}-r_{\mathrm{i}}\right\}$. Also shown in Fig. 1 are DMC ground-state total energies per hole for $2 \mathrm{~h}^{+}$, $\mathrm{X}, \mathrm{X}^{-}, \mathrm{X}^{+}$, and $\mathrm{XX}$, all of which are bound. These confirm that the ground-state energies of the single-hole complexes $\left(\mathrm{X}\right.$ and $\mathrm{X}^{-}$) are very close to the energy of a single hole, while the ground-state energies of the twohole complexes $\left(\mathrm{X}^{+}\right.$and $\left.\mathrm{XX}\right)$ are comparable with the energy of two confined holes. The ground-state energies for single- and two-hole complexes vary slightly differently as a function of aspect ratio due to the interaction between the holes. The capacitive charging energy $E_{\mathrm{CC}}=E^{2 \mathrm{~h}^{+}}-2 E^{\mathrm{h}^{+}}$for the experimentally relevant ring geometry [20] is $E_{\mathrm{CC}}=8.8546(8) \mathrm{meV}$; this compares to an experimentally measured value [34] of $E_{\mathrm{CC}}=24(2)$ meV. STM images of quantum rings [20, 21] suggest that the GaSb/GaAs interface is not clean in practice. This disorder could lead to trapping of holes, strongly affecting capacitive charging energies while having relatively little effect on binding energies.

The de-excitonization energies for the trions and biexciton in the geometry modeling the quantum rings described in Ref. 20 can be found in Table I. The deexcitonization energy is positive for $\mathrm{X}^{-}$, but negative for $\mathrm{X}^{+}$and $\mathrm{XX}$. The negative de-excitonization energy is a result of the large energy penalty when two holes are confined to the same ring; e.g., two excitons on two separate quantum rings would be the energetically preferred fourparticle state rather than a biexciton on a single ring. The expected positions of these peaks in a photoluminescence spectrum are shown in Fig. 2. The $\mathrm{X}^{-}$peak is very close to the $\mathrm{X}$ peak, while the peaks for $\mathrm{X}^{+}$and $\mathrm{XX}$ are separated from the $\mathrm{X}$ peak by a few $\mathrm{meV}$. The heights of the peaks indicate the relative stability of the complexes, using binding energy data from Table I] Experimental work has not yet progressed to the point where excitonic complex peak positions have been identified. The only published work showing sharp lines in the photoluminescence spectra of GaSb/GaAs quantum rings is Ref. [21]; however the spectra in this work would likely contain

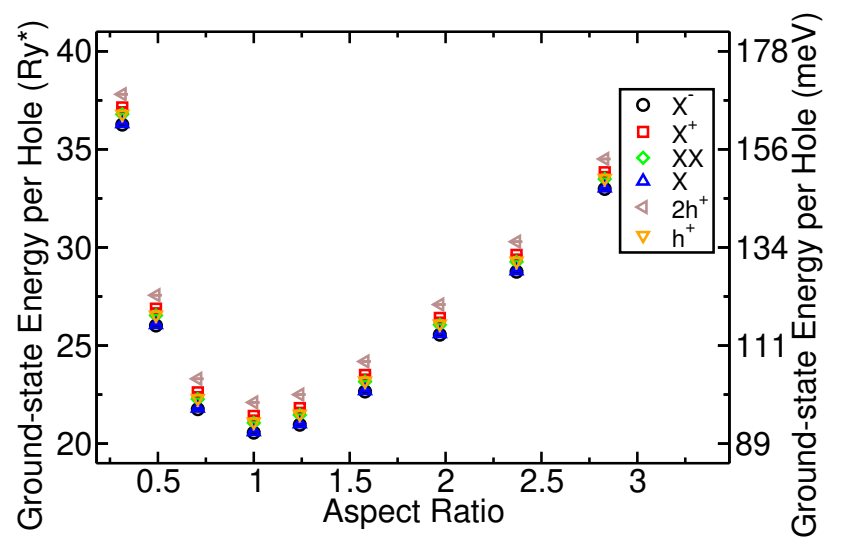

FIG. 1. Ground-state total energies per hole of a single hole $\left(\mathrm{h}^{+}\right)$, two holes $\left(2 \mathrm{~h}^{+}\right)$, an exciton $(\mathrm{X})$, a negative trion $\left(\mathrm{X}^{-}\right)$, a positive trion $\left(\mathrm{X}^{+}\right)$, and a biexciton $(\mathrm{XX})$ in a quantum ring plotted against the aspect ratio $2 R_{z} /\left(r_{\mathrm{o}}-r_{\mathrm{i}}\right)$ of the ring's cross section. The mean radius and ring volume are appropriate for the GaSb/GaAs quantum rings reported in Ref. 20]. Error bars are smaller than the size of the symbols. The exciton Rydberg $R_{\mathrm{y}}^{*}$ is $4.45 \mathrm{meV}$ for the experimentally relevant geometry.

TABLE I. De-excitonization $E_{\mathrm{D}}$ and binding $E_{\mathrm{b}}$ energies for excitonic complexes in the quantum-ring geometry modeling the samples described in Ref. 20].

\begin{tabular}{lcccc}
\hline \hline Complex & $E_{\mathrm{D}} / R_{\mathrm{y}}^{*}$ & $E_{\mathrm{b}} / R_{\mathrm{y}}^{*}$ & $E_{\mathrm{D}}(\mathrm{meV})$ & $E_{\mathrm{b}}(\mathrm{meV})$ \\
\hline $\mathrm{X}$ & 0 & $-0.5004(6)$ & 0 & $-2.226(3)$ \\
$\mathrm{X}^{-}$ & $+0.0446(4)$ & $-0.0446(4)$ & $+0.199(2)$ & $-0.199(2)$ \\
$\mathrm{X}^{+}$ & $-1.111(2)$ & $-1.379(2)$ & $-4.944(7)$ & $-6.137(7)$ \\
$\mathrm{XX}$ & $-0.911(2)$ & $-0.701(2)$ & $-4.052(8)$ & $-3.11(1)$ \\
\hline \hline
\end{tabular}

peaks from many, highly positively charged rings, making a direct comparison with theoretical values difficult. The de-excitonization energy is plotted against the aspect ratio of the cross-section of the ring for $\mathrm{X}^{-}, \mathrm{X}^{+}$, and $\mathrm{XX}$ in Fig. 3(a). For each complex it can be seen that there is some slight change in the de-excitonization energy as a function of aspect ratio. The de-excitonization energies are largely independent of the aspect ratio, and hence exact shape of the ring, somewhat justifying the use of a ring with a rectangular cross-section in our model. Furthermore, the energetic effects of the slight interpenetration of the electron and hole orbitals are likely to be well described by a slight renormalization of the cross-section of the ring; however the effects of such small changes in the cross-section appear to be small.

The binding energies for each complex are shown in Table II for the experimentally relevant geometry [20]. The $\mathrm{X}$ binding energy is about half the value for a free $\mathrm{X}$ due to the exclusion of the electron from the ring. As expected, $\mathrm{X}^{-}$is the most weakly bound (against dissociation into a free electron and a neutral exciton), while $\mathrm{X}^{+}$is the most stable (against removal of an electron from a ring of charge of $+2 e)$. From these binding en- 


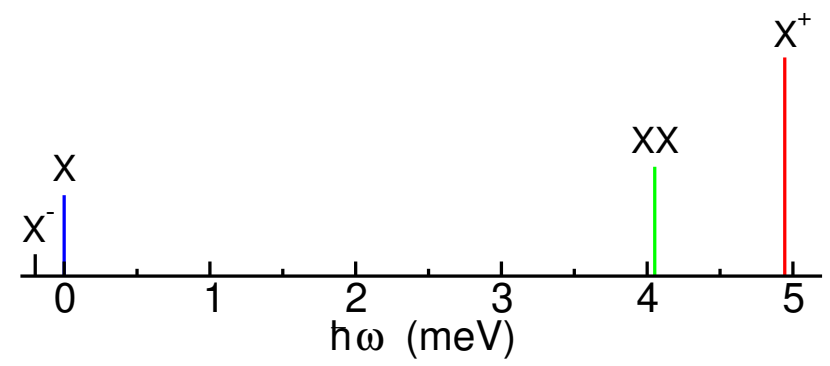

FIG. 2. Expected peak positions for the excitonic complexes in a photoluminescence spectrum relative to the exciton peak, for a model of the quantum rings reported in Ref. 20. The peak heights represent the relative stability of the complexes.

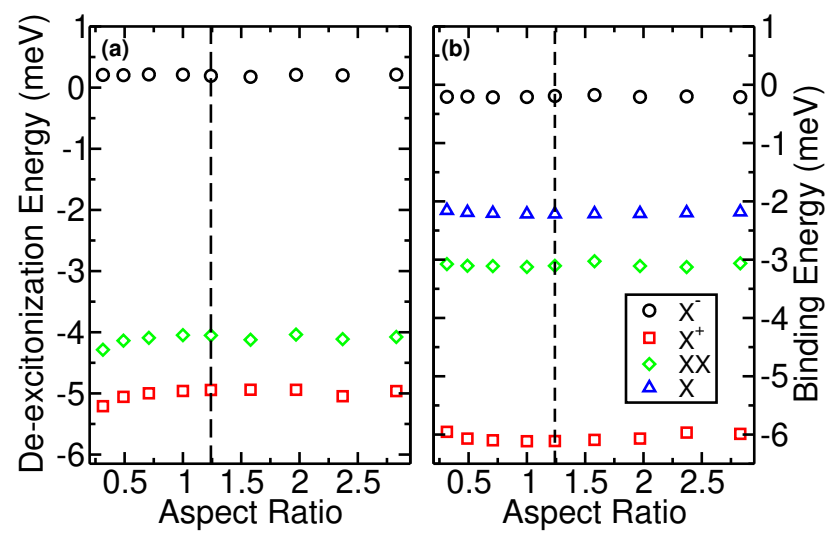

FIG. 3. (a) De-excitonization energies and (b) binding energies against the aspect ratio $2 R_{z} /\left(r_{\mathrm{o}}-r_{\mathrm{i}}\right)$ of a quantum ring's cross-section for different charge-carrier complexes. The mean radius and ring volume are appropriate for the GaSb/GaAs quantum rings reported in Ref. [20]. Error bars are smaller than the size of the symbols. The dashed lines shows the experimentally relevant aspect ratio [20].

ergies the temperatures up to which the complexes are stable are $26,2.3,71$, and $36 \mathrm{~K}$ for $\mathrm{X}, \mathrm{X}^{-}, \mathrm{X}^{+}$, and $\mathrm{XX}$, respectively. As with the de-excitonization energies, the binding energy depends weakly on the aspect ratio of the ring's cross-section, but again these differences are much smaller than the differences in binding energy between complexes: see Fig. 3(b). Therefore, the binding energy appears to be largely independent of the exact shape of the cross-section of the ring for a given ring volume and mean radius.

Plots of the electronic charge density for each complex in the experimentally relevant geometry are shown in Fig. 4. The electrons form a diffuse halo around the ring, with negligible charge density in the ring's central cavity. The kinetic-energy cost of localizing in the ring's cavity significantly exceeds the gain in electrostatic potential energy. Correlation effects further reduce the probability of finding multiple electrons inside the ring's cavity. XX and $\mathrm{X}^{+}$are the most localized complexes, as reflected in their relatively large binding energies shown in Table I. These two-hole complexes have slightly higher electronic charge
TABLE II. Sensitivity of the biexciton binding energy to the electron and hole effective masses $m_{\mathrm{e}}$ and $m_{\mathrm{h}}$, the relative permittivity $\epsilon$, the ring volume $V$, and the mean radius of the ring $r_{\mathrm{m}}$.

\begin{tabular}{ccccc}
\hline $\begin{array}{l}\partial E_{\mathrm{b}}^{\mathrm{xx}} / \partial m_{\mathrm{e}} \\
\left(\mathrm{meV} / m_{\mathrm{e}}\right)\end{array}$ & $\begin{array}{l}\mathrm{x} E_{\mathrm{b}}^{\mathrm{Xx}} / \partial m_{\mathrm{h}} \\
\left(\mathrm{meV} / m_{\mathrm{e}}\right)\end{array}$ & $\begin{array}{c}\partial E_{\mathrm{b}}^{\mathrm{Xx}} / \partial \epsilon \\
(\mathrm{meV})\end{array}$ & $\begin{array}{c}\partial E_{\mathrm{b}}^{\mathrm{Xx}} / \partial V \\
\left(\mathrm{meV} / \mathrm{nm}^{3}\right)\end{array}$ & $\begin{array}{c}\partial E_{\mathrm{b}}^{\mathrm{Xx}} / \partial r_{\mathrm{m}} \\
(\mathrm{meV} / \mathrm{nm})\end{array}$ \\
\hline$-7.4(3)$ & $-0.20(4)$ & $0.39(1)$ & $0.0004(2)$ & $0.07(2)$ \\
\hline \hline
\end{tabular}

densities in the regions directly above and below the center of the ring compared to the regions to the left and right of the ring. STM images of the electronic density of states in Ref. [20] suggest the electrons are localized to the ring's cavity, which does not agree with the results presented here. However, in the STM experiments the sample is cleaved in the $x-z$ plane. This is a drastic modification to the system, which is not described by our model. It is plausible that the reduced screening and hence smaller free exciton Bohr radius in the cleaved system allows electrons to localize within, rather than above or below, the quantum ring.

The sensitivity of the XX binding energy to various parameters is presented in Table II. The XX binding energy depends most strongly on the electron effective mass, and is relatively insensitive to the hole effective mass, relative permittivity, ring volume, and mean ring radius. Our conclusions are robust against reasonable uncertainties in model parameters.

Kehili et al. 35. have recently investigated excitons in GaSb rings in GaAs quantum wells using the effectivemass approximation, modeling the ring with a finite potential, and including strain effects due to latticeconstant mismatch. In their work the interaction between charge carriers is described by a Hartree mean-field approximation, in contrast to the complete treatment of

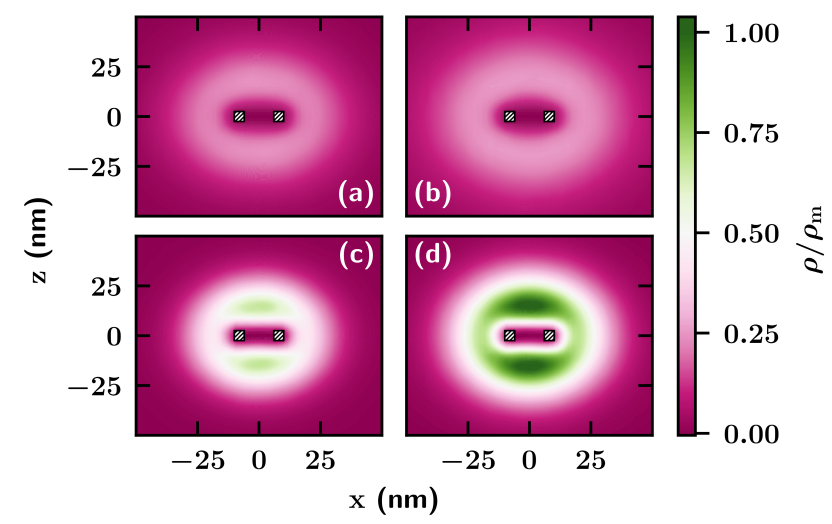

FIG. 4. Electronic charge density $\rho$ for (a) an exciton, (b) a negative trion, (c) a positive trion, and (d) a biexciton in the experimentally relevant quantum-ring geometry [20]. The shaded regions represent the ring and $\rho_{m}$ is the maximum density across all four plots. The free exciton Bohr radius is $a_{0}^{*}=12.5 \mathrm{~nm}$. 
correlation effects used here. Nevertheless, their electronic charge density is qualitatively consistent with our results. Their X binding is slightly larger than our value reported in Table II] however, partly due to their use of slightly different effective masses and mean ring radii. A DMC calculation of the $\mathrm{X}$ binding energy using the same ring geometry and effective masses as Kehili et al. gives $E_{\mathrm{b}}^{\mathrm{X}}=2.695(2) \mathrm{meV}$, which is comparable with the binding energy of about $2.6 \mathrm{meV}$ that they report for a GaAs well of width of $40 \mathrm{~nm}$ (the largest well width they consider). The $\mathrm{X}$ binding energies reported by Kehili et $a l$. do not appear to have converged with respect to well width at this point, however, and it looks as if they will be significantly smaller than the DMC exciton binding energy in the limit of large well width. This is consistent with the fact that, by the variational principle, Hartree theory underestimates the magnitude of the $\mathrm{X}$ binding energy.

\section{CONCLUSION}

In conclusion, total energies of excitonic complexes in type-II quantum-ring heterostructures are dominated by the confinement energy of the holes in each complex. The de-excitonization energy is positive for $\mathrm{X}^{-}$as would be the case for a free trion; however, for $\mathrm{X}^{+}$and $\mathrm{XX}$ this en- ergy is negative due to the energy penalty associated with confining two holes in the same ring. $\mathrm{X}^{-}$is the least stable of the complexes studied; it is predicted to be stable only at temperatures below $2.3 \mathrm{~K}$, while the most stable complex, $\mathrm{X}^{+}$, endures up to $71 \mathrm{~K}$. De-excitonization and binding energies were shown to be largely independent of the aspect ratio at fixed ring volume and mean radius, suggesting these energies may also be fairly independent of the precise shape of the cross-section of the ring. The electrons form a halo around the outside of the ring, with a low density in the central cavity. This reflects the fact that the ring size is comparable with the free exciton Bohr radius. Furthermore, $\mathrm{X}^{+}$and $\mathrm{XX}$ are the most tightly bound complexes, with a preference for the electrons to position themselves above and below the ring. For $\mathrm{X}^{-}$, the electronic charge density is much more diffuse, consistent with its very small binding energy.

\section{ACKNOWLEDGMENTS}

D.M.T. and R.J.H. are fully funded by the Graphene NOWNANO CDT (EPSRC Grant No. EP/L01548X/1). Computer resources were provided by Lancaster University's High End Computing Cluster. We acknowledge useful discussions with B. Garikipati.
[1] R. C. Ashoori, Nature 379, 413 (1996).

[2] D. Gammon, Nature 405, 899 (2000).

[3] R. J. Warburton, C. Schäflein, D. Haft, F. Bickel, A. Lorke, K. Karrai, J. M. Garcia, W. Schoenfeld, and P. M. Petroff, Nature 405, 926 (2000)

[4] M. Bayer, O. Stern, P. Hawrylak, S. Fafard, and A. Forchel, Nature 405, 923 (2000).

[5] U. Banin, Y. W. Cao, D. Katz, and O. Millo, Nature 400, 542 (1999)

[6] A. Fuhrer, S. Lüscher, T. Ihn, T. Heinzel, K. Ensslin, W. Wegscheider, and M. Bichler, Nature 413, 822 (2001)

[7] F. Nagasawa, D. Frustaglia, H. Saarikoski, K. Richter, and J. Nitta, Nat. Commun. 4, 2526 (2013)

[8] B. Bansal, M. Hayne, M. Geller, D. Bimberg, and V. V. Moshchalkov, Phys. Rev. B 77, 241304 (2008).

[9] B. Bansal, S. Godefroo, M. Hayne, G. Medeiros-Ribeiro, and V. V. Moshchalkov, Phys. Rev. B 80, 205317 (2009)

[10] M. Kroutvar, Y. Ducommun, D. Heiss, M. Bichler, D. Schuh, G. Abstreiter, and J. J. Finley, Nature 432, $81(2004)$

[11] T. Nowozin, A. Marent, L. Bonato, A. Schliwa, D. Bimberg, E. P. Smakman, J. K. Garleff, P. M. Koenraad, R. J. Young, and M. Hayne, Phys. Rev. B 86, 035305 (2012)

[12] M. Hayne, J. Maes, S. Bersier, V. V. Moshchalkov, A. Schliwa, L. Müller-Kirsch, C. Kapteyn, R. Heitz, and D. Bimberg, Appl. Phys. Lett. 82, 4355 (2003)

[13] K. Suzuki, R. A. Hogg, and Y. Arakawa, J. Appl. Phys. 85, 8349 (1999)
[14] F. Hatami, M. Grundmann, N. N. Ledentsov, F. Heinrichsdorff, R. Heitz, J. Böhrer, D. Bimberg, S. S. Ruvimov, P. Werner, V. M. Ustinov, P. S. Kopev, and Z. I. Alferov, Phys. Rev. B 57, 4635 (1998)

[15] M. Geller, C. Kapteyn, L. Müller-Kirsch, R. Heitz, and D. Bimberg, Appl. Phys. Lett. 82, 2706 (2003).

[16] R. B. Laghumavarapu, A. Moscho, A. Khoshakhlagh, M. El-Emawy, L. F. Lester, and D. L. Huffaker, Appl. Phys. Lett. 90, 173125 (2007)

[17] A. J. Williamson, L. W. Wang, and A. Zunger, Phys. Rev. B 62, 12963 (2000).

[18] A. D. Andreev and E. P. O'Reilly, Phys. Rev. B 62, 15851 (2000).

[19] P. J. Carrington, R. J. Young, P. D. Hodgson, A. M. Sanchez, M. Hayne, and A. Krier, Cryst. Growth and Des. 13, 1226 (2013).

[20] R. J. Young, E. P. Smakman, A. M. Sanchez, P. Hodgson, P. M. Koenraad, and M. Hayne, Appl. Phys. Lett. 100, $082104(2012)$

[21] M. P. Young, C. S. Woodhead, J. Roberts, Y. J. Noori, M. T. Noble, A. Krier, E. P. Smakman, P. M. Koenraad, M. Hayne, and R. J. Young, AIP Adv. 4, 117127 (2014)

[22] P. D. Hodgson, M. Hayne, A. J. Robson, Q. D. Zhuang, and L. Danos, J. Appl. Phys. 119, 044305 (2016)

[23] R. Timm, H. Eisele, A. Lenz, L. Ivanova, V. Vossebürger, T. Warming, D. Bimberg, I. Farrer, D. A. Ritchie, and M. Dähne, Nano Lett. 10, 3972 (2010).

[24] M. Hayne, R. J. Young, E. P. Smakman, T. Nowozin, P. Hodgson, J. K. Garleff, P. Rambabu, P. M. Koenraad, A. Marent, L. Bonato, A. Schliwa, and D. Bimberg, J. 
Phys. D: Appl. Phys. 46, 264001 (2013)

[25] S. Kobayashi, C. Jiang, T. Kawazu, and H. Sakaki, Jpn. J. Appl. Phys. 43, L662 (2004)

[26] D. M. Ceperley and B. J. Alder, Phys. Rev. Lett. 45, 566 (1980).

[27] W. M. C. Foulkes, L. Mitas, R. J. Needs, and G. Rajagopal, Rev. Mod. Phys. 73, 33 (2001).

[28] R. J. Needs, M. D. Towler, N. D. Drummond, and P. López Ríos, J. Phys. Condens. Matter 22, 023201 (2010)

[29] T. Kato, Commun. Pure Appl. Math. 10, 151 (1957)
[30] N. D. Drummond, M. D. Towler, and R. J. Needs, Phys. Rev. B 70, 235119 (2004).

[31] P. López Ríos, P. Seth, N. D. Drummond, and R. J. Needs, Phys. Rev. E 86, 036703 (2012).

[32] C. J. Umrigar, J. Toulouse, C. Filippi, S. Sorella, and R. G. Hennig, Phys. Rev. Lett. 98, 110201 (2007)

[33] M. E. Levinshten, S. L. Rumyantsev, and M. Shur, 1 , Vol. 1 (World Scientific, 1996).

[34] P. D. Hodgson, R. J. Young, M. Ahmad Kamarudin, P. J. Carrington, A. Krier, Q. D. Zhuang, E. P. Smakman, P. M. Koenraad, and M. Hayne, J. Appl. Phys. 114, 073519 (2013)

[35] M. S. Kehili, A. Ben Mansour, R. Sellami, and A. Melliti, Semicond. Sci. Technol. 33, 115019 (2018). 